\title{
Unexpected relationship between fat mass and basal metabolic rate in pregnant women
}

\author{
BY MICHELE N. BRONSTEIN, ROSA P. MAK AND JANET C. KING* \\ Department of Nutritional Sciences, University of California at Berkeley, Berkeley, CA 94720, USA
}

(Received 18 October 1993 - Revised 4 August 1995 - Accepted 15 September 1995)

\begin{abstract}
We investigated the relationships between BMR, fat-free mass (FFM) and fat mass in pregnancy. BMR was measured by indirect calorimetry and body composition was assessed by densitometry in seventeen non-pregnant women (79.9 (SD 26-3, range 50.5-151.4) kg) and sixteen pregnant women (75.7 (SD 20.6, range 54.5-115.9) kg). The pregnant women were evaluated during weeks 31-35 of gestation. Multiple regression analysis of BMR with FFM and fat mass in the non-pregnant women showed that FFM was a highly significant predictor of BMR $(P<0.0001)$, but fat mass was not $(P=0.09)$. In contrast, in the pregnant women, multiple regression analysis revealed that fat mass was a highly significant predictor $(P<0.001)$, while FFM was not $(P=0.69)$. Evaluation of the interaction terms in the combined data set confirmed that the relationships of BMR with FFM and fat mass differ significantly in non-pregnant and pregnant women. It is proposed that pregnancy represents a unique condition during which $B M R$ is regulated by maternal adipose reserves. An augmented BMR in overweight pregnant women may be protective, given that excessive weight gain may be detrimental to neonatal and maternal health.
\end{abstract}

Pregnancy: Basal metabolic rate: Body composition

Basal metabolism is an excellent predictor of total daily energy expenditure in pregnant women (Prentice et al. 1989; Goldberg et al. 1993). Recent work suggests, however, that there is tremendous interindividual variability in the magnitude of the change in basal metabolism during pregnancy (Forsum et al. 1988; Prentice et al. 1989; Goldberg et al. 1993). Given that variability, it is important to identify the factors that influence basal metabolism during pregnancy. This knowledge may assist in individualizing energy recommendations for pregnant women.

In non-pregnant women, fat-free mass (FFM) is the single best predictor of BMR, regardless of differences in body composition (Ravussin \& Bogardus, 1989; Cunningham, 1991). Few studies have carefully examined the relationships between the components of body mass and BMR in pregnant women. The most frequent finding has been that while BMR increases on an absolute basis $(\mathrm{kJ} / \mathrm{d})$ from mid to late pregnancy, there is no difference or only a slight difference between non-pregnant and pregnant values when BMR data are expressed on either a per kg body mass or a per kg FFM basis (Nagy \& King, 1983; Prentice et al. 1989; van Raaij et al. 1989; Goldberg et al. 1993). This observation has led to the belief that the metabolic activity of tissue acquired during pregnancy is similar to that of non-pregnant tissue, and that weight or lean-tissue gain is the primary determinant of the change in BMR during pregnancy. This understanding has been disputed by the findings of a study which showed that absolute changes in BMR over the course of pregnancy were not well correlated with the amount of lean tissue acquired (Prentice et al. 1989). Instead, changes in basal metabolism appeared to be related more to prepregnancy energy status. The authors speculated that gestation may represent a special

\footnotetext{
* For reprints.
} 
state during which metabolic rate is particularly flexible, and that this flexibility is regulated by prepregnancy maternal energy stores. In support of this theory, undernourished pregnant women from the Gambia show a depression in BMR when expressed per kg FFM (Poppitt et al. 1993), and it is thought that this decline in BMR may be an energy-sparing adaptation in the undernourished (Lawrence et al. 1987).

To date, no one has examined how a prepregnancy surplus of adipose stores changes basal metabolism during pregnancy. If metabolic rate is truly responsive to maternal energy status during pregnancy, one might expect a greater increase in the BMR of women who commence pregnancy overweight. In this report we present BMR data measured in sixteen third trimester pregnant women, eight of whom were overweight. Similar data were collected in seventeen non-pregnant women for comparison. Eight of the non-pregnant women were overweight. The relationships between BMR, FFM, fat mass and body weight are presented for both pregnant and non-pregnant women.

\section{METHODS}

\section{Subjects}

Nine normal-weight non-pregnant, eight overweight non-pregnant, eight normal-weight pregnant and eight overweight pregnant women aged 20-35 years participated in this study. Overweight was defined as a BMI $\geqslant 25 \mathrm{~kg} / \mathrm{m}^{2}$. Pregnant women were classified according to their prepregnancy weight, which was determined by recall at time of recruitment and confirmed by medical records when available. All women were assessed to be in good health before acceptance for study. The women were non-smokers and were not taking any medications. Only sedentary women with fewer than three children were accepted. An acceptable recreational activity level included any one of the following: aerobics $\leqslant 1$ time/week, jogging $<5$ miles/week, swimming $\leqslant 1 \mathrm{~h} /$ week, and cycling $\leqslant 1 \mathrm{~h} /$ week. Non-pregnant women selected were weight stable and not recent (within 6 months) oralcontraceptive users. A $3 \mathrm{~d}$ weighed diet record was utilized to exclude women who engaged in dieting $(<5051 \mathrm{~kJ} / \mathrm{d} ;<1200 \mathrm{kcal} / \mathrm{d})$ excessive overeating $(>12552 \mathrm{~kJ} / \mathrm{d}$; $>3000 \mathrm{kcal} / \mathrm{d}$ ) or extreme dietary habits. Overweight women were additonally selected based on a lifelong and family history of being overweight, and difficulty in losing weight through energy restriction.

Each woman was given a detailed description of the study protocol and gave her informed consent. This study was approved by the Committees on the Use and Protection of Human Subjects at the University of California at Berkeley and the University of California, San Francisco.

\section{$B M R$}

All energy expenditure measurements were performed at the General Clinical Research Center (GCRC) at the San Francisco General Hospital. BMR was measured three times in each subject. The first measurement served to adapt the subjects to the energy expenditure equipment and testing procedures. Reported BMR values represent the mean of the latter two measurements. For the pregnant women the latter two measurements were performed during weeks $31-35$ of gestation and were separated by a 2-week period. BMR in the non-pregnant women was evaluated during the follicular phase of their menstrual cycle and the two measurements were separated by a 4-week period. Before both test days subjects adhered to a $4 \mathrm{~d}$ take-home diet, the energy content of which equalled 1.38 times the resting energy expenditure level determined on the first test day (Prentice et al. 1985). The function of the take-home diet was to normalize subject energy intake before testing procedures. 
For the latter two measurements of BMR, subjects were admitted to the GCRC in the evening and were awakened at 06.30 hours the following morning. On awakening, the subject voided and returned to bed to lie quietly for $30 \mathrm{~min}$ before testing. BMR was measured by open-circuit, indirect calorimetry using the Douglas bag technique. Subjects were fitted with noseclips and mouthpieces, and breath gas was collected for three 8 min periods after allowing 2 min for adaptation to the breathing apparatus. Expired gas volume was monitored using a pneumoscan (KL Engineering, Sylimar, CA, USA) positioned before the Douglas bag. On emptying the Douglas bag, gas volume was rechecked using a gasometer (Singer Corporation, American Meter Division, Elizabeth, NJ, USA), and a small gas sample was passed over a Drierite (WH Hammond Drierite Co., Xenia, OH, USA) column and then collected into an airtight, Al-coated mylar bag for later analysis. The $\mathrm{O}_{2}$ and $\mathrm{CO}_{2}$ contents of the gas sample were analysed by an electrochemical $\mathrm{O}_{2}$ analyser (Model S-3A, Applied Electrochemistry, Inc. Sunnyvale, CA, USA) and an infrared $\mathrm{CO}_{2}$ analyser (LB-2, Beckman Instruments, Anaheim, $\mathrm{CA}$ and Ametek Instruments, Pittsburgh, PA, USA). Gas analysers were calibrated before use with room air and reference standards. Resting metabolic rate was calculated from $\mathrm{O}_{2}$ and $\mathrm{CO}_{2}$ consumption and $\mathrm{CO}_{2}$ production measurements according to the equation of Weir (1949).

\section{Body composition assessment}

Body fat content was determined by densitometry. Pregnant women were measured 1 month postpartum when it was assumed that the hydration of the FFM had returned to the non-pregnant state. Densitometry was performed by underwater displacement. Subjects donned standardized bathing suits and were lowered into a calibrated tank of water at $37^{\circ}$. While the subject was immersed underwater, lung volume was estimated by the He dilution technique. Gastrointestinal volume was assumed to be $0 \cdot 1$ litres. Body volume measurements were performed in triplicate. Body density was calculated from body weight/body volume, and percentage body fat was derived from body density using the equation of Siri (1956)

$$
\% \text { fat }=100 \times(4 \cdot 95 / \text { body density })-4 \cdot 5 \text {. }
$$

Fat mass was determined from total body weight multiplied by percentage fat.

For the pregnant women it was assumed that fat mass measured at 1 month postpartum was representative of fat mass at 31-35 weeks of gestation. This assumption was based on research showing little maternal fat deposition during the last 2 months of gestation (Hytten \& Chamberlain, 1980; Forsum et al. 1988; Goldberg et al. 1993) and negligible loss of maternal fat tissue during the first month postpartum (Sadurskis et al. 1988). FFM at study (i.e. 31-35 weeks gestation) in the pregnant women was calculated from the difference between total body weight at study and fat mass at 1 month postpartum. Densitometry measurements could not be performed for three pregnant subjects. For two of these subjects body fat mass was estimated from skinfold determinations made 1 month postpartum. For the third subject, body fat mass and FFM were estimated from a determination of total body water made at the time of the BMR measurement. Total body water was estimated by ${ }^{18} \mathrm{O}$ dilution (Schoeller et al. 1980). FFM was estimated from total body water by assuming that FFM contains $75.9 \%$ water at 31-35 weeks of gestation (Seitchik, 1967); body fat mass was derived from the difference between body weight and FFM. For the skinfold method, four sites (biceps, triceps, subscapular and suprailiac) were measured in triplicate by the same individual using calibrated Lange calipers (Lange, Springfield, MA, USA). An average of the three measurements was taken, and percentage body fat was calculated according to established equations (Durnin \& Rahaman, 1967). 


\section{Statistical analysis}

Statistical analyses were performed using the Statistical Package for the Social Sciences (SPSS Inc., Chicago, Illinois, USA). Subject profile and mean BMR values for the nonpregnant and pregnant women were compared using Student's unpaired $t$ test. Pearson's correlation coefficient was computed to assess the reproducibility of BMR measurements. Simple linear and multiple regression analyses were performed to examine the relationships between BMR and FFM, fat mass, and body weight for the non-pregnant and pregnant women separately. Simultaneous multiple regression analysis with interaction terms (pregnancy $\times$ FFM ; pregnancy $\times$ fat mass) was then performed on the combined BMR data set to evaluate whether there was a significant effect of pregnancy on the relationship between BMR and FFM or BMR and fat mass. This later analysis included the independent variables FFM, fat mass, pregnancy status and the interaction terms (pregnancy $\times$ FFM) and (pregnancy $\times$ fat mass). All values are expressed as means and standard deviations except where otherwise noted. For all statistical analyses the level of significance was defined as $P<0.05$.

\section{RESULTS}

A profile of the subject population is presented in Table 1. All subjects were Caucasian, except two non-pregnant subjects who were African-American. Non-pregnant women did not differ from pregnant women with respect to any of the anthropometric and body composition characteristics.

The measurement of BMR was highly reproducible as the correlation coefficient for the duplicate measurements was $0.97(P<0.001)$. Intraindividual variability was minimal, the CV were 1.8 and $2.5 \%$ for the non-pregnant and pregnant women respectively. BMR, expressed per unit time $(\mathrm{MJ} / \mathrm{d})$, was significantly $(P<0.001)$ greater in the pregnant women (Table 1).

Simple linear regression analyses of BMR with the variables FFM, fat mass, and body weight in the non-pregnant women revealed significant correlations with FFM ( $r$ 0.90; $P<0.0001)$, fat mass $(r 0.80 ; P=0.0001)$ and body weight $(r 0.89 ; P<0.0001)$. In the pregnant women BMR was significantly correlated with fat mass $(r 0.93, P<0.0001)$ and body weight $(r 0.88, P<0.0001)$. Unexpectedly, in the pregnant women, BMR was not significantly correlated with FFM $(r 0.37, P=0 \cdot 15)$. The relationship between BMR and FFM, fat mass and body weight in the pregnant women did not change on omission of the data from the three pregnant women whose body composition was assessed by alternative techniques. Fig. 1 illustrates the simple regression of BMR $v$. FFM in the non-pregnant and pregnant women separately. While the results of the simple linear regression analyses were noteworthy, the univariate equations generated by these analyses are of limited usefulness as they do not allow one to look at the independent effects of each variable upon BMR in the presence of the others. Multiple regression analysis was thus employed to evaluate simultaneously the relative importance of FFM and fat mass as predictors of BMR. The results of the multiple regression analysis, in which the non-pregnant and pregnant data sets were evaluated separately, are presented in Table 2. For the nonpregnant group, when both FFM and fat mass were in the regression FFM was highly statistically significant, but fat mass was not. In contrast, fat mass was a highly significant predictor of BMR in the pregnant women but FFM was not. It can be observed from the coefficients with their standard errors in Table 2 that the relationships of FFM and fat mass with BMR were very different in the non-pregnant and pregnant groups. To examine this issue in a more rigorous fashion we performed a multiple regression analysis on the 
Table 1. Subject profile and basal metabolic characteristics

(Mean values and standard deviations, with ranges in parentheses)

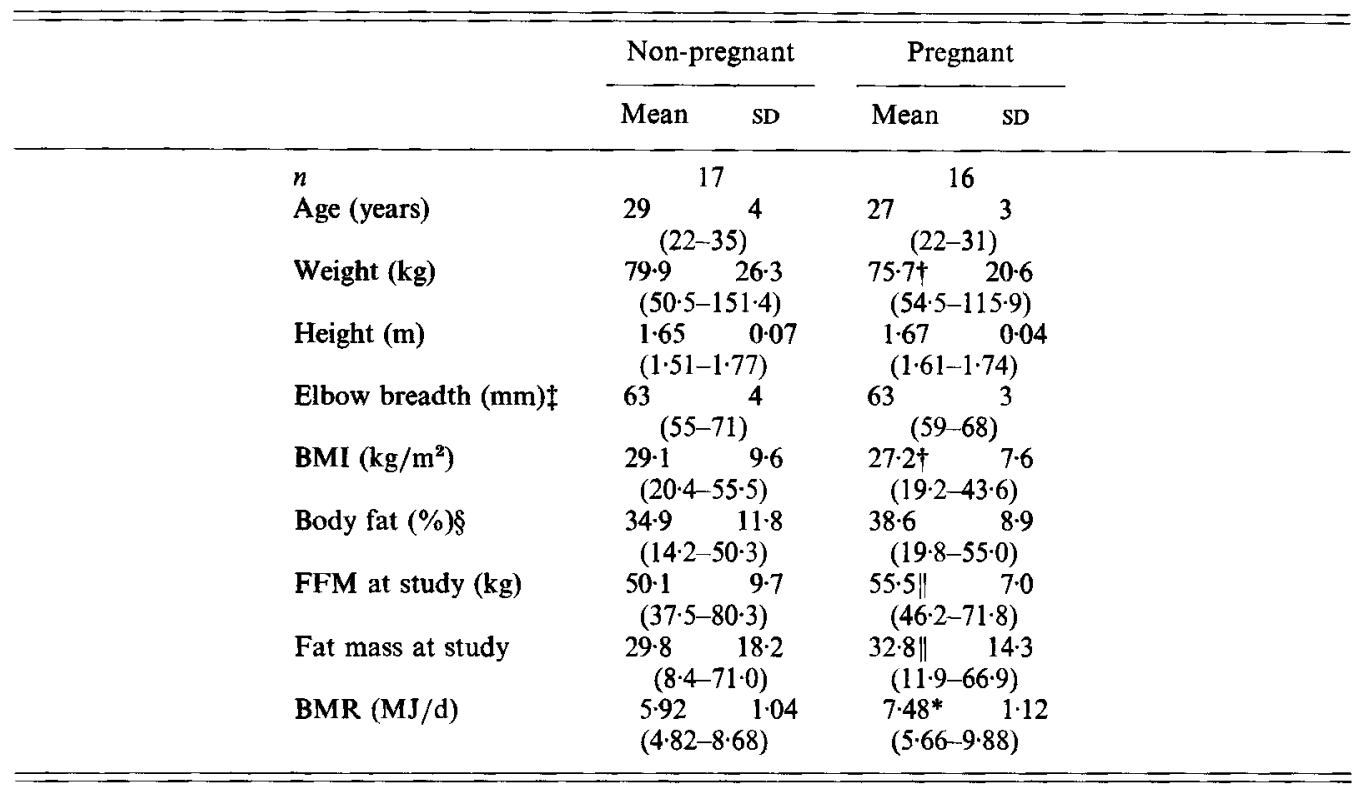

FFM, fat-free mass.

* Mean value was significantly different from that for non-pregnant women, $P<0.05$.

$\dagger$ Based on reported pregravid weight.

¥ Elbow breadth is an indicator of skeletal frame size.

$\S$ Percentage body fat was determined by densitometry, except for three subjects. Skinfolds were used to assess postpartum body fat for two pregnant studies. Values are missing for one pregnant subject. Percentage body fat was determined at study in non-pregnant women and 1 month postpartum for the pregnant women.

|| Based on measurements performed 1 month postpartum, except for one pregnant subject. For this subject FFM and fat mass at study (31-35 weeks gestation) were determined from a measurement of total body water made at 33 weeks gestation. For further details, see p. 661.
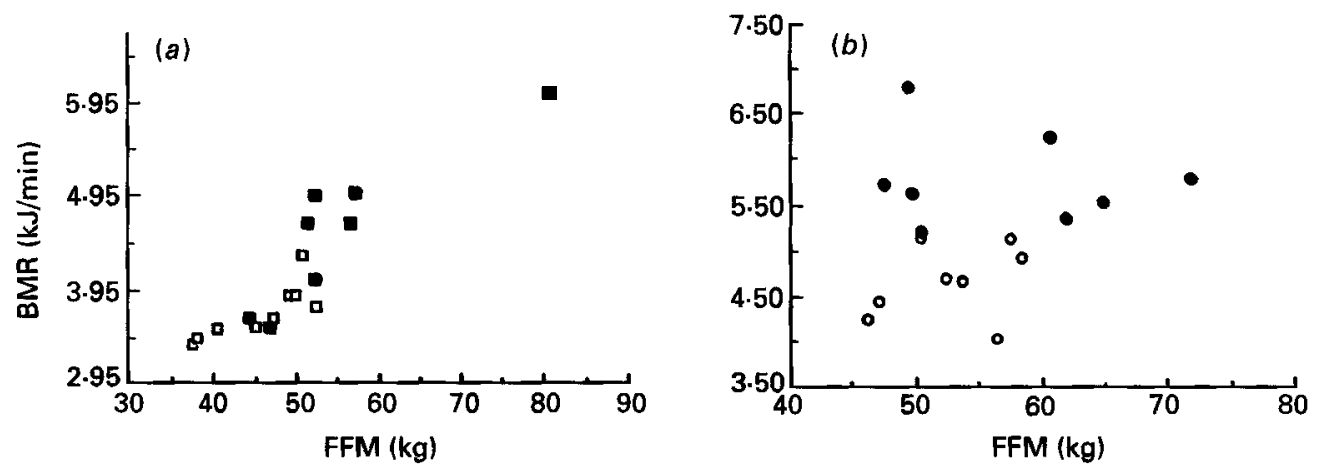

Fig. 1. Relationship between BMR (kJ/min) and (a), fat-free mass (FFM; kg) in non-pregnant women ( $r 0.90$, $P<0.0001)$ and $(b)$, FFM in pregnant women $(r 0.37$, NS). Measurements of BMR in the pregnant women were performed during weeks 31-35 of gestation. ( $\square$ ), Normal-weight non-pregnant; $(\square)$, overweight non-pregnant; $(\bigcirc)$, normal-weight pregnant; (O), overweight pregnant women. 
Table 2. Multiple regression equations for prediction of $B M R(k J / d)$ from fat-free mass (FFM) and fat mass in non-pregnant and pregnant women*

(Coefficients with their standard errors)

\begin{tabular}{|c|c|c|c|c|c|c|}
\hline & \multicolumn{3}{|c|}{$\begin{array}{c}\text { Non-pregnant } \\
(n 17)\end{array}$} & \multicolumn{3}{|c|}{$\begin{array}{l}\text { Pregnant } \\
(n 16)\end{array}$} \\
\hline & Coefficient & $\mathbf{S E}$ & $P$ & Coefficient & SE & $P$ \\
\hline Constant & 1707 & 648 & & 4740 & 893 & \\
\hline FFM & $\mathbf{7 4 . 9 7}$ & $16 \cdot 14$ & $<0.001$ & 7.04 & $17 \cdot 16$ & 0.69 \\
\hline Fat mass & $15 \cdot 82$ & $8-57$ & 0.086 & $71 \cdot 36$ & $8 \cdot 48$ & $<0.001$ \\
\hline$r^{2}$ & $0 \cdot 86$ & & & 0.87 & & \\
\hline
\end{tabular}

* Regression equation: $B M R=$ constant $+\left(b_{1} \times F F M\right)+\left(b_{2} \times\right.$ fat mass $)$.

Table 3. Evaluation of the effect of pregnancy on the relationship between $B M R$ and fat-free mass $(F F M)$ or $B M R$ and fat mass using multiple regression analysis*

(Analysis was performed on data collected from seventeen non-pregnant and sixteen pregnant women)

\begin{tabular}{lcrl}
\hline & Coefficient & SE & $P$ \\
\hline Constant & 1707 & 666 & \\
Pregnancy $(0=$ non-pregnant, $1=$ pregnant $)$ & 3034 & 1095 & \\
FFM & 74.97 & 16.57 & \\
Fat mass & 15.82 & 8.80 & \\
Pregnancy $\times$ FFM & -67.93 & 23.53 & 0.008 \\
Pregnancy $\times$ fat mass & 55.55 & 12.07 & 0.0001 \\
$r^{2}$ & 0.91 & & \\
\hline
\end{tabular}

* Multiple regression analysis included the independent variables FFM, fat mass, pregnancy status and the interaction terms (pregnancy $\times$ FFM) and (pregnancy $\times$ fat mass).

combined data set (Table 3). This analysis included FFM, fat mass, pregnancy status, and the interaction terms (pregnancy $\times$ FFM) and (pregnancy $\times$ fat mass). Both interaction terms were statistically significant $(P=0.008$ and $P<0.0001$ respectively), implying that the relationships of FFM and fat mass with BMR were different for the non-pregnant and pregnant groups.

\section{DISCUSSION}

Body size and composition are the primary determinants of basal metabolism. FFM is thought to be the most important body compartment related to BMR. In fact, subject differences in basal expenditure per unit time can typically be normalized by expressing BMR on a per kg FFM basis. Our results suggest, however, that the relationship between BMR and FFM is modified in late pregnancy.

Multiple regression analysis of the dependent variable BMR with the independent variables FFM and fat mass in the seventeen non-pregnant women showed that FFM was highly significant and fat mass was not (Table 2). This result is consistent with those of other research groups (Cunningham, 1991). However, when a similar analysis was performed for the sixteen pregnant women, fat mass was highly significant, whereas FFM was not (Table 2). Furthermore, multiple regression analysis on the combined data set 
confirmed that these relationships differed significantly between non-pregnant and pregnant women.

A possible criticism of the present study is the reliability of these body composition measurements performed in pregnant women with a wide range of fat mass. The body fat of the pregnant women at the time of study (31-35 weeks gestation) was estimated from densitometry measurements performed 1 month postpartum. We assumed that maternal fat stores did not change appreciably during the latter 5-9 weeks of pregnancy and during the first month postpartum. While previous research suggests our assumptions were appropriate (Hytten \& Chamberlain, 1980; Forsum et al. 1988; Sadurskis et al. 1988; Goldberg et al. 1993), they may have introduced error in our estimate of body composition in the pregnant women. The presence of excessive fat stores may also have introduced error, as half of our study population was overweight. Womersley et al. (1976) have suggested that excess body fat leads to an underestimation of FFM by densitometry.

A possible explanation for the significant relationship between fat mass and BMR observed in the pregnant women in the present study is that maternal energy status plays a role in regulating basal metabolism during pregnancy. Prentice et al. (1989) observed that the change in BMR during pregnancy correlated with prepregnancy maternal fatness, not lean tissue. In their study, the prepregnancy percentage body fat of nearly all the subjects was not excessive (11.5-31.0\% prepregnant fat); only one woman was markedly obese ( $40.8 \%$ prepregnant fat) Interestingly, the incremental increase in basal metabolism was highest in this obese woman. Our results, which include the study of eight normal-weight and eight overweight pregnant women, provide further support for the role of maternal body fat in regulating basal expenditure during pregnancy, and suggest that the overweight pregnant woman is energy-profligate (Fig. 2). On average, the eight overweight pregnant women evaluated in the present investigation expended 8.34 (SD 0.85) MJ/d (range $7.41-9.88 \mathrm{MJ} / \mathrm{d}$ ) for basal metabolism compared with 6.61 (SD 0.62) $\mathrm{MJ} / \mathrm{d}$ (range $5 \cdot 66-7 \cdot 35 \mathrm{MJ} / \mathrm{d}$ ) for the eight normal-weight pregnant women studied.

An ability to lower energy expenditure in pregnant women with low maternal fat reserves would be of great value in the developing world where the demands of work are high and access to food is limited. In malnourished Gambian women the cumulative change in resting metabolism during pregnancy decreased (Lawrence et al. 1987; Poppitt et al. 1993). When a group of Gambian women were given food supplements the cumulative change in resting metabolism during pregnancy rose to $4 \mathrm{MJ}$ (Lawrence et al. 1987). In contrast, the cumulative metabolic cost in well-nourished women ranges from 126 to $194 \mathrm{MJ}$ (Committee on Nutritional Status During Pregnancy and Lactation, Food and Nutrition Board, Institute of Medicine, 1990). These results suggest that BMR is particularly flexible and sensitive to maternal energy status during pregnancy, and that it is markedly depressed in women with low fat stores.

At the other end of the spectrum, our results show that BMR is enhanced among pregnant women with high fat stores. This ability to increase expenditure may limit gestational weight gain in overweight women. In contrast to findings in underweight and normal-weight women, gestational weight gain in overweight women has little or no impact on birth weight (Abrams \& Laros, 1986; Mitchell \& Lerner, 1987; Frentzen et al. 1988). There are several potential advantages to limiting gestational weight gain in overweight women. Perinatal mortality rates among overweight women are lowest for a weight gain of $7.3 \mathrm{~kg}$, compared with $9.1 \mathrm{~kg}$ for normal-weight women and $13.6 \mathrm{~kg}$ for underweight women (Naeye, 1979). The fetus may be sensitive to nutritional excesses, as well as nutritional deficiencies (Naeye, 1979). The overweight woman herself is at increased risk of pregnancy-induced hypertension and gestational diabetes (Gross et al. 1980). If excessive weight gain jeopardizes the health status of either the fetus or mother with excessive fat 

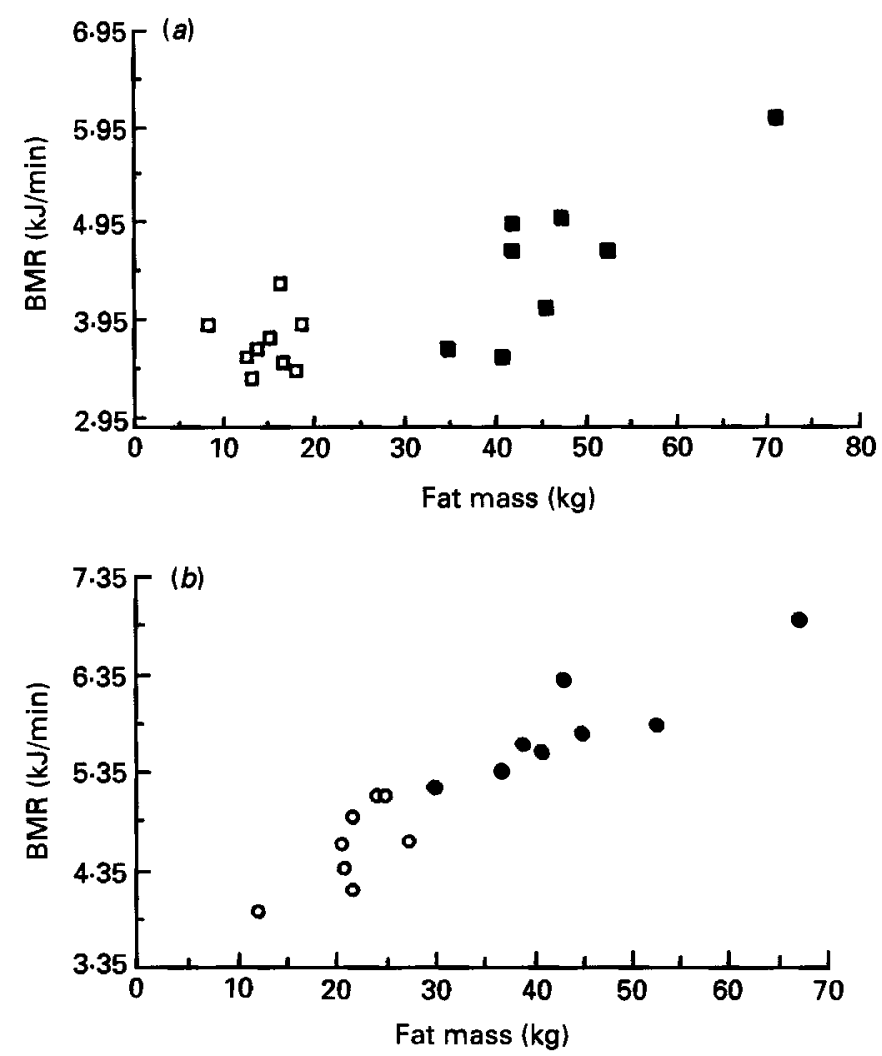

Fig. 2. Relationship between BMR $(\mathrm{kJ} / \mathrm{min})$ and $(a)$, fat mass $(\mathrm{kg})$ in non-pregnant women $(r 0.80, P<0.0001)$ and $(b)$, fat mass in pregnant women $(r 0.93, P<0.0001)$. Measurements of BMR in the pregnant women were performed during weeks 31-35 of gestation. ( $\square$ ), Normal-weight non-pregnant; $(\square)$, overweight non-pregnant; $(\bigcirc)$, normal-weight pregnant; (O), overweight pregnant women.

stores, an 'energy wasting' mechanism which limits weight gain could be extremely valuable.

There is other evidence which suggests that energy expenditure is increased in overweight pregnant women. They tend to gain less weight on average than normal-weight pregnant women; some even lose weight (Abrams \& Laros, 1986; Frentzen et al. 1988; Committee on Nutritional Status During Pregnancy and Lactation, Food and Nutrition Board, Institute of Medicine, 1990). Low weight gains have occurred even when overweight women were counselled to increase their rates of weight gain (Abrams \& Laros, 1986; Frentzen et al. 1988). Normal-weight women appear to gain weight readily during pregnancy without significant changes in energy intake (Committee on Nutritional Status During Pregnancy and Lactation, Food and Nutrition Board, Institute of Medicine, 1990), whereas overweight women do not. A tempting explanation for this observation is that the metabolic demands of pregnancy are greater in overweight women than in normal weight women.

Findings showing a decline in birth weight with increasing maternal fat mass in overweight women also support the hypothesis that maternal metabolic costs rise with progressive obesity, diverting energy from the fetus. Luke \& Petrie (1980) found that infant birth weight tended to drop as postpartum body weight rose within the range of 160 to $210 \%$ of standard weight for height. Similarly, Kleinman (Committee on Nutritional 
Status During Pregnancy and Lactation, Food and Nutrition Board, Institute of Medicine, 1990) found an increased incidence of low birth weight in full-term infants of women with a $\mathrm{BMI}>26$ and total weight gain exceeding $15 \mathrm{~kg}$. These observations suggest that fetal growth is compromised with increasing adiposity in overweight women, possibly because maternal metabolic demands are increased. These findings do not explain, however, why overweight women tend to have larger infants. One possible explanation is that the increased fetal size is related to the greater FFM of overweight women. Several studies have shown that fetal growth rates are linked to maternal lean body mass, not fat mass (Briend, 1985; Langhoff-Roos et al. 1987; Lawrence et al. 1991). On average, the eight overweight pregnant women in the present study had a larger frame size (66 (SD 2) v. 61 (SD 1) mm elbow breadth; $P<0.05$ ) and greater FFM (57 (SD 8.5) v. 52.8 (SD 4.6) $\mathrm{kg} ; P<0.05$ ) than did the eight normal-weight pregnant women. Also the overweight pregnant women had heavier (4.0 (SD 0.4) v. 3.3 (SD 0.3) kg; $P<0.05$ ) and longer (554 (SD 25) v. 508 (SD 10) mm; $P<0.05)$ infants than did the normal-weight pregnant women, even though the normalweight pregnant women gained slightly more weight $(14.0$ (SD 4.9) v. 17.4 (SD 7.0) $\mathrm{kg} ; P=$ $0 \cdot 14)$.

Prior studies showing that energy is conserved in the presence of maternal thinness (Poppitt et al. 1993) and the results of the present study showing an energy-wasting phenomenon with maternal obesity suggest a unique role for maternal fat reserves during gestation. For underweight women a low fat reserve may down-regulate maternal energy metabolism to support the gain of lean tissue and fat stores. A certain threshold of adipose reserves may be necessary as an energy buffer for the mother during late pregnancy and to provide an energy reserve for lactation. Once this body fat level is achieved, however, additional maternal fat gain is unnecessary and may even be detrimental, especially if it is excessive. We postulate that excessive maternal fat reserves trigger an increase in maternal energy expenditure and protect against the unhealthy acquisition of additional adipose tissue. Perhaps the hormones of pregnancy are sensitive to maternal energy status and are responsible for this flexibility in energy metabolism.

In conclusion, the findings of this investigation show that the relationships between BMR, FFM and fat mass are altered during pregnancy. Pregnancy seems to be a state of metabolic flexibility that is influenced by maternal adipose reserves. Longitudinal studies of overweight pregnant women are needed to confirm this conclusion and would improve our understanding of the dietary requirements for overweight pregnant women.

This project was supported in part by USDA grant no. 85-CRCR-1-1553 and the General Clinical Research Center, San Francisco General Hospital, NIH grant no. 5T32 HD07266, and NIH grant no. RR-00083.

\section{REFERENCES}

Abrams, B. F. \& Laros, R. K. (1986). Prepregnancy weight, weight gain, and birth weight. American Journal of Obstetrics and Gynecology 154, 503-509.

Briend, A. (1985). Do maternal energy reserves limit fetal growth? Lancet $\mathbf{i}, 38-40$.

Committee on Nutritional Status During Pregnancy and Lactation, Food and Nutrition Board, Institute of Medicine (1990) Nutrition During Pregnancy. Washington, DC: National Academy Press.

Cunningham, J. J. (1991). Body composition as a determinant of energy expenditure: a synthetic review and a proposed general prediction equation. American Journal of Clinical Nutrition 54, 963-969.

Durnin, J. V. G. A. \& Rahaman, M. M. (1967). The assessment of the amount of fat in the human body from measurements of skinfold thickness. British Journal of Nutrition 21, 681-689.

Forsum, E., Sadurskis, A. \& Wager, J. (1988). Resting metabolic rate and body composition of healthy Swedish women during pregnancy. American Journal of Clinical Nutrition 47, 942-947.

Frentzen, B. H., Dimperio, D. L. \& Cruz, A. C. (1988). Maternal weight gain: effect on infant birth weight among overweight and average-weight low-income women. American Journal of Obstetrics and Gynecology 159, 1114-1117. 
Goldberg, G. R., Prentice, A. M., Coward, W. A., Davies, H. L., Murgatroyd, P. R., Wensing, C., Black, A. E., Harding, M. \& Sawyer, M. (1993). Longitudinal asseessment of energy expenditure in pregnancy by the doubly labeled water method. American Journal of Clinical Nutrition 57, 494-505.

Gross, T., Sokol, R. J. \& King, K. C. (1980). Obesity in pregnancy: risks and outcome. Obstetrics and Gynecology 56, 446-450.

Hytten, F. \& Chamberlain, G. (1980). Clinical Physiology in Obstetrics. Oxford: Blackwell Scientific Publications.

Langhoff-Roos, J., Lindmark, G. \& Gebre-Medhin, M. (1987). Maternal fat stores and fat accretion during pregnancy in relation to infant birthweight. British Journal of Obstetrics and Gynaecology 94, 1170-1177.

Lawrence, M., Lawrence, F., Coward, W. A., Cole, T. J. \& Whitehead, R. G. (1987). Energy requirements of pregnancy in the Gambia. Lancet ii, 1072-1076.

Lawrence, M., McKillop, F. M. \& Durnin, J. V. G. A. (1991). Women who gain more fat during pregnancy may not have bigger babies: implications for recommended weight gain during pregnancy. British Journal of Obstetrics and Gynaecology 98, 254-259.

Luke, B. \& Petrie, R. H. (1980). Intrauterine growth: correlation of infant birth weight and maternal postpartum weight. American Journal of Clinical Nutrition 33, 2311-2317.

Mitchell, M. C. \& Lerner, E. (1987). Factors that influence the outcome of pregnancy in middle-class women. Journal of the American Dietetics Association 87, 731-735.

Naeye, R. L. (1979). Weight gain and the outcome of pregnancy. American Journal of Obstetrics and Gynecology 135, 3-9.

Nagy, L. E. \& King, J. C. (1983). Energy expenditure of pregnant women at rest or walking self-paced. American Journal of Clinical Nutrition 38, 369-376.

Poppitt, S. D., Prentice, A. M., Jéquier, E., Schutz, Y. \& Whitehead, R. G. (1993). Evidence of energy sparing in Gambian women during pregnancy: a longitudinal study using whole-body calorimetry. American Journal of Clinical Nutrition 57, 353-364.

Prentice, A. M., Coward, W. A., Davies, H. L., Murgatroyd, P. R., Black, A. E., Goldberg, G. R., Ashford, J., Sawyer, M. \& Whitehead, R. G. (1985). Unexpectedly low levels of energy expenditure in healthy women. Lancet i, $1419-1422$.

Prentice, A. M., Goldberg, G. R., Davies, H. L., Murgatroyd, P. R. \& Scott, W. (1989). Energy-sparing adaptations in human pregnancy assessed by whole-body calorimetry. British Journal of Nutrition 62, 5-22.

Ravussin, E. \& Bogardus, C. (1989). Relationship of genetics, age, and physical fitness to daily energy expenditure and fuel utilization. American Journal of Clinical Nutrition 49, 968-975.

Sadurskis, A., Kabir, N., Wager, J. \& Forsum, E. (1988). Energy metabolism, body composition, and milk production in healthy Swedish women during lactation. American Journal of Clinical Nutrition 48, 44-49.

Schoeller, D. A., van Santen, E., Peterson, D. W., Dietz, W., Jaspan, J. \& Klein, P. D. (1980). Total body water measurement in humans with ${ }^{18} \mathrm{O}$ and ${ }^{2} \mathrm{H}$ labeled water. American Journal of Clinical Nutrition 33, $2686-2693$.

Seitchik, J. (1967). Total body water and total body density of pregnant women. Obstetrics and Gynecology 29, $155-166$.

Siri, W. E. (1956). Gross composition of the body. In Advances in Biological and Medical Physics, vol. 4, pp. 239-280 [J. H. Lawrence and C. A. Tobias, editors]. New York: Academic Press.

van Raaij, J. M. A., Schonk, C. M., Vermaat-Miedema, S. H., Peek, M. E. M. \& Hautvast, J. G. A. J. (1989). Body fat mass and basal metabolic rate in Dutch women before, during and after pregnancy: a reappraisal of energy cost of pregnancy. American Journal of Clinical Nutrition 49, 765-772.

Weir, J. B. de V. (1949). New methods for calcufating metabolic rate with special reference to protein metabolism. Journal of Physiology 109, 1-9.

Womersley, J., Durnin, J. V. G. A., Boddy, K. \& Mahaffy, M. (1976). Influence of muscular development, obesity, and age on the fat-free mass of adults. Journal of Applied Physiology 41, 223-228. 